\title{
Integrated FDG-PET/CT imaging is useful in the approach to carcinoid tumors of the lung
}

\author{
Alessandro Stefani ${ }^{1 *}$, Antonella Franceschetto ${ }^{2}$, Jessica Nesci ${ }^{1}$, Beatrice Aramini ${ }^{1}$, Chiara Proli ${ }^{1}$, Shaniko Kaleci ${ }^{3}$, \\ Alessandra Casolo ${ }^{2}$, Lucia Massi ${ }^{2}$, Christian Casali ${ }^{1}$ and Uliano Morandi ${ }^{1}$
}

\begin{abstract}
Background: Carcinoids enter the differential diagnosis of the solitary pulmonary nodule. Bronchial carcinoids have been traditionally considered as FDG-PET negative but recent studies have found an higher sensitivity of integrated FDG-PET/CT for the detection of these neoplasms. The purpose of this study was to investigate the value of integrated FDG-PET/CT for the evaluation of SPN suspected to be carcinoids.

Methods: All patients with pathologically proven bronchial carcinoids who had FDG-PET/CT scans between 2006 and 2012 have been retrospectively reviewed. PET/CT was performed with the same scanner and the same technique for all patients. The following data were retrieved: age, sex CT findings (side, location, size, shape, margins), SUVmax, type of operation, pathological findings (size and number of mitoses). Regarding PET findings, only SUVmax was considered, whereas the visual assessment was not undertaken. Carcinoids were defined as typical and atypical and as central and peripheral. The long-term follow-up was also recorded. The SUVmax was compared with the other clinical, radiological and pathological variables to find any significant difference or correlation.

Results: Twenty-five patients were retrieved, 24 typical and one atypical carcinoid, 21 peripheral and 4 central lesions. The mean diameter on CT-scan was $25.3 \mathrm{~mm}$ and the clinical size correlated well with the pathological size. Sixty percent of the tumors were ovoid and 68\% had smooth margins. The mean SUVmax was 3.6 (range 1.4-12.9). All the lesions were completely resected. The regression analysis showed a direct correlation between the SUVmax and the tumor size $(p=0.004)$. No further correlations were found between the SUVmax and the other variables. None of the patients had recurrent disease or died during the follow-up.

Conclusions: Our study showed that FDG-PET/CT might be a useful tool in the evaluation of SPNs suspected to be bronchial carcinoids. When a solitary pulmonary nodule shows an ovoid/round shape and smooth margins on the CT scan and demonstrates an FDG uptake higher than that of the normal lung and with a SUVmax value $>1-1.5$, a carcinoid should be suspected. If benign lesions can be presumably excluded, surgical resection or at least a biopsy of the lesion is recommended.
\end{abstract}

Keywords: Bronchial carcinoid, Positron emission tomography, Standardized uptake value

\section{Background}

Bronchial carcinoids account for 1-2\% of lung neoplasms [1]. They are histologically classified as typical (low-grade malignancies, accounting for $85-90 \%$ of all carcinoids) and atypical (intermediate-grade) [2]. Eighty percent of pulmonary carcinoids are endobronchial in origin and they can be easily diagnosed by fiberoptic bronchoscopy biopsy,

\footnotetext{
* Correspondence: alessandro.stefani@unimore.it

'Department of Thoracic Surgery, University of Modena and Reggio Emilia, Modena, Italy

Full list of author information is available at the end of the article
}

whereas $20 \%$ occur peripherally and manifest as solitary nodules or masses on chest imaging, usually in asymptomatic patients [3]. These peripheral forms are part of the differential diagnosis of solitary pulmonary nodule (SPN). Imaging with somatostatin-receptor scintigraphy could be used in cases of a suspected carcinoid $[4,5]$ but, when an SPN is found on a computed tomography (CT) scan, many diseases other than carcinoid can be suspected, such as lung carcinoma, hamartoma, inflammatory or infectious lesions. Thus, in clinical practice, somatostatinreceptor scintigraphy is not the examination of choice

\section{Biomed Central}

(c) 2013 Stefani et al.; licensee BioMed Central Ltd. This is an open access article distributed under the terms of the Creative Commons Attribution License (http://creativecommons.org/licenses/by/2.0), which permits unrestricted use, distribution, and reproduction in any medium, provided the original work is properly cited. 
after CT in the evaluation of SPN. 18 F-fluorodeoxyglucose positron emission tomography (FDG-PET) is the preferred examination in this setting [6]. More recently, integrated FDG-PET/CT has been shown to be an effective tool to differentiate malignant from benign lesions [7]. But carcinoids, especially in the typical form, show a low metabolic activity which reduces the sensitivity of FDG-PET for the diagnosis of these neoplasms [8]. In fact, bronchial carcinoids have been traditionally considered FDG-PET negative and this understanding led many physicians to consider this technique as not a valuable tool for the diagnosis of these tumors. Conversely, recent studies have found a higher sensitivity of FDG-PET for the detection of carcinoids, for both the typical and atypical forms [9-11], although these studies have reported series with a limited number of patients. Therefore, peripheral carcinoids presenting as SPNs remain a diagnostic challenge.

The purpose of this study was to investigate the value of integrated FDG-PET/CT for the evaluation of bronchial carcinoids by reviewing our institutional experience since 2006.

\section{Methods}

Patients

We retrospectively reviewed all the patients with pathologically proven carcinoid tumors who had FDG-PET/ CT scans at our institution, between January 2006 and December 2012.

The study was approved by the Institutional Review Board of the University of Modena and Reggio Emilia.

At our institution FDG-PET/CT scanning was introduced in 2006 and has been used in the preoperative diagnostic and staging work-up of patients with SPN suspected to be lung tumors. The following data were retrieved from the clinical records of patients affected by carcinoid who entered the study: age, sex, CT findings, FDG-PET/CT findings, preoperative diagnosis (if available), type of operation, number of mitoses and surgical stage. Central carcinoid was defined if the tumor was visible on the bronchoscopy examination, otherwise the tumor was defined as peripheral, regardeless of its location within the lung parenchyma. When the tumor was visible on bronchoscopy, an endoscopic biopsy of the lesion was undertaken.

Patients were referred to surgery because of the suspicion of a malignant lesion, predominantly based on CT and PET findings. Enhanced CT-scans were acquired with helical technique in all patients, before FDG-PET/CT examination. The indication to perform an FDG-PET/CT was based on the results of CT scan. All patients underwent surgical resection and the final diagnosis was based on the histopathology of the surgical specimens. Lateral muscle-sparing thoracotomy was the surgical approach. Surgical resection was lobectomy or limited resection in all cases and mediastinal lymph node dissection was routinely performed. A frozen section examination was undertaken in all the cases without preoperative diagnosis.

\section{FDG-PET/CT}

All the FDG-PET/CT scans were reviewed by nuclear medicine experts, who were not blinded.

Whole-body FDG-PET/CT was performed with a combined PET/CT scanner (GE Discovery DSTE, General Electric medical systems, Milwaukee, WI, USA). This scanner allows multi-detector row helical CT scanning with 16 array of detectors. The technical parameters used for the CT were as follows: a detector row configuration of 4 sections of $3.75 \mathrm{~mm}$ thickness, pitch of $1.375: 1, \mathrm{KVp}: 120$, a gantry rotation speed of $0.8 \mathrm{~s}$ and an amperage of $80-250 \mathrm{~mA}$, modulated to maintain a noise index of 30, in free breath. The CT examination was used for attenuation correction of PET images.

The patients fasted for at least 6 hours prior to the scan. After verification of serum glucose levels of $\leq 150 \mathrm{mg} / \mathrm{dl}$, the patients received an intravenous injection of $3.7 \mathrm{MBq} / \mathrm{Kg}$ of FDG. The scans were started 60 to 70 minutes after the injection and scanned the area from the base of the brain to the mid-thigh. The emission PET scan was obtained with a $2.5 \mathrm{~min}$ acquisition per bed position; typically, six or seven bed positions are obtained. The scanner operates in the three-dimensional mode and the images were reviewed in axial, coronal and sagittal formats. For this study, visual analysis was not performed and we considered only the tumor activity using the maximum standardized uptake value (SUVmax), to minimize the partial volume effect on the uptake value. The SUVmax is a semiquantitative measurement of tissue metabolic rate and represents the maximum measured activity at the region of interest (ROI), placed manually over the most intense area of FDG accumulation, on the axial attenuation corrected PET images.

CT images of both helical CT scan and integrated PET/ CT were analyzed for the tumor location, tumor size, shape and margin characteristics, presence of calcifications, necrotic low-attenuation areas and associated postobstructive atelectasis or pneumonia. The tumor location was expressed in terms of side and lobe and the tumor size was defined by the longest diameter. The tumor shape was classified as ovoid, round or lobulated and the tumor margins as smooth or spiculated.

\section{Pathology}

Typical carcinoids (TC) and atypical carcinoids (AC) were defined on the basis of the current World Health Organization criteria as follows [12]: TC has $<2$ mitotic figures per $2 \mathrm{~mm}^{2}$ and no necrosis, whereas $\mathrm{AC}$ has 2 to 10 mitotic figures per $2 \mathrm{~mm}^{2}$ or evidence of necrosis. 
The presence of lymph node metastases was reported and expressed as N1 (hilar) or N2 (mediastinal).

\section{Survival}

The patients were routinely followed up at our institution and the survival data were available in our clinical databases. Follow-up was updated on May 2013 for all patients.

\section{Statistical analysis}

The objective of the study was to investigate the accuracy of FDG-PET/CT in the evaluation of bronchial carcinoids. The SUVmax of all the lesions was calculated and compared with the other clinical, radiological and pathological variables, to find any significant difference or correlation.

The statistical analysis was conducted using the SPSS ${ }^{\circ}$ statistical software (Chicago, Ill, USA). Data were expressed in terms of frequencies, mean and standard deviation (SD). The comparisons were performed with the chi-square test for categorical variables and the t-test for continuous variables. The correlation between SUVmax and size was investigated using Spearman's correlation coefficient and the goodness-of-fit measure of each linear model $\left(R^{2}\right)$ was reported. A p value of less than 0.05 was considered significant.

\section{Results}

Twenty-five patients presenting with SPN, having FDGPET/CT and undergoing subsequent surgical resection for suspected malignancy were found to have bronchial carcinoids.

The patients characteristics are presented in Table 1. There were 22 women (88\%) and 3 men, and mean age was 58.8 years (range 29-78). No tumors showed features of ectopic hormonal secretion (carcinoid or Cushing syndrome). The left lung was involved in 14 cases (56\%); the left upper lobe and right middle lobe were most frequently involved ( 9 and 8 cases, respectively). Most of the lesions were located peripherally (21/25,

Table 1 Clinical characteristics and PET/CT findings of the 25 patients

\begin{tabular}{|c|c|c|c|c|c|c|c|c|c|c|c|}
\hline \multirow{2}{*}{ Pts } & \multirow{2}{*}{ age } & \multirow{2}{*}{ sex } & \multicolumn{5}{|c|}{ CT findings } & \multirow{2}{*}{$\begin{array}{l}\text { PET findings } \\
\text { SUVmax }\end{array}$} & \multirow{2}{*}{$\begin{array}{l}\text { Surgical } \\
\text { procedure }\end{array}$} & \multicolumn{2}{|c|}{ Histologic findings } \\
\hline & & & Lobe location & Lesion location & Size $(\mathrm{mm})$ & Shape & Margin & & & Cell type & No of mitoses \\
\hline 1 & 78 & $\mathrm{~F}$ & LUL & Peripheral & 12 & ovoid & smooth & 1.9 & Lobectomy & Typical & 1 \\
\hline 2 & 62 & $\mathrm{~F}$ & LUL & Peripheral & 18 & ovoid & smooth & 4.6 & Lobectomy & Typical & 1 \\
\hline 3 & 64 & $\mathrm{~F}$ & RML & Peripheral & 20 & lobulated & smooth & 12.9 & Lobectomy & Typical & 1 \\
\hline 4 & 29 & $\mathrm{~F}$ & LLL & Central & 70 & ovoid & spiculated & 9.7 & Lobectomy & Typical & 1 \\
\hline 5 & 36 & $\mathrm{~F}$ & RML & Peripheral & 28 & lobulated & spiculated & 4.2 & Lobectomy & Typical & 1 \\
\hline 6 & 67 & $\mathrm{~F}$ & RML & peripheral & 15 & ovoid & spiculated & 2.8 & Lobectomy & Typical & 1 \\
\hline 7 & 74 & $\mathrm{~F}$ & LUL & Peripheral & 18 & ovoid & spiculated & 2.4 & Wedge resection & Typical & 1 \\
\hline 8 & 51 & $\mathrm{~F}$ & RML & Peripheral & 15 & ovoid & smooth & 1.9 & Lobectomy & Typical & 1 \\
\hline 9 & 49 & $\mathrm{~F}$ & RML & Peripheral & 25 & lobulated & smooth & 6.0 & Lobectomy & Typical & 1 \\
\hline 10 & 61 & M & LUL & Peripheral & 23 & ovoid & smooth & 1.5 & Lobectomy & Typical & 1 \\
\hline 11 & 61 & M & LUL & Central & 42 & ovoid & smooth & 2.2 & Lobectomy & Typical & 1 \\
\hline 12 & 51 & $\mathrm{~F}$ & LUL & Peripheral & 23 & lobulated & spiculated & 3.0 & Lobectomy & Typical & 1 \\
\hline 13 & 61 & $\mathrm{~F}$ & LLL & Peripheral & 20 & ovoid & smooth & 4.5 & Segmentectomy & Typical & 0 \\
\hline 14 & 35 & $\mathrm{~F}$ & LLL & Peripheral & 38 & lobulated & smooth & 5.0 & Lobectomy & Atypical & 4 \\
\hline 15 & 60 & $\mathrm{~F}$ & LLL & Central & 55 & round & spiculated & 4.2 & Lobectomy & Typical & 1 \\
\hline 16 & 72 & $\mathrm{~F}$ & RUL & Peripheral & 24 & round & smooth & 3.1 & Lobectomy & Typical & 1 \\
\hline 17 & 47 & $\mathrm{~F}$ & LLL & Central & 40 & ovoid & smooth & 2.0 & Lobectomy & Typical & 1 \\
\hline 18 & 63 & $\mathrm{~F}$ & RML & Peripheral & 14 & lobulated & smooth & 2.4 & Lobectomy & Typical & 1 \\
\hline 19 & 70 & M & LUL & Peripheral & 23 & ovoid & spiculated & 6.2 & Wedge resection & Typical & 0 \\
\hline 20 & 61 & $\mathrm{~F}$ & RUL & Peripheral & 13 & lobulated & smooth & 1.4 & Lobectomy & Typical & 0 \\
\hline 21 & 60 & $\mathrm{~F}$ & RUL & Peripheral & 15 & ovoid & smooth & 1.5 & Wedge resection & Typical & 1 \\
\hline 22 & 60 & $\mathrm{~F}$ & RML & Peripheral & 18 & lobulated & smooth & 1.8 & Wedge resection & Typical & 1 \\
\hline 23 & 78 & $\mathrm{~F}$ & LUL & Peripheral & 16 & ovoid & smooth & 1.8 & Lobectomy & Typical & 1 \\
\hline 24 & 61 & $\mathrm{~F}$ & RML & Peripheral & 34 & ovoid & spiculated & 3.1 & Lobectomy & Typical & 1 \\
\hline 25 & 61 & $\mathrm{~F}$ & LUL & Peripheral & 15 & ovoid & smooth & 1.7 & Lobectomy & Typical & 1 \\
\hline
\end{tabular}


84\%). The mean diameter on the CT-scan was $25.3 \mathrm{~mm}$ (range 12-70, SD 14 mm), and most tumors were ovoid $(\mathrm{n}=15,60 \%)$ and had smooth margins $(\mathrm{n}=17,68 \%)$. Necrotic areas, calcifications, atelectasis or obstructive pneumonia were not found in any case.

The mean SUVmax of the nodules was 3.6 and the median SUVmax was 2.8 (range 1.4-12.9, SD 2.7). The FDG-PET did not reveal significant uptake in hilar or mediastinal lymph nodes, and the CT scan was negative for suspected node metastases in all cases.

Tissue diagnosis was obtained by bronchoscopic biopsy, prior to surgical resection, in the 4 patients with central lesions (16\%); in these cases a diagnosis of suspected carcinoid was achieved. In the other patients the diagnosis was obtained by frozen section examination and confirmed on histopathological analysis.

All the lesions were completely resected, in most cases by lobectomy $(20 / 25,80 \%)$. The final diagnoses revealed $24 \mathrm{TCs}$ and one AC. The mean pathological size was $24 \mathrm{~mm}$ (range 7-68, SD 14.9) and the pathological dimensions were comparable with the clinical dimensions $(p=0.239)$. All the TCs showed one or no mitoses per $2 \mathrm{~mm}^{2}$, whereas the AC showed 4 mitoses per $2 \mathrm{~mm}^{2}$. The patient with AC had one metastatic hilar lymph node (N1) whereas the other cases were free of lymph node involvement.

All the variables were compared and the differences are as follows: regarding age and location, the patients with central lesions were younger than those with peripheral carcinoids (mean age 47 and 62 years respectively, $\mathrm{p}=0.033$ ); concerning side and location, all the central lesions were on the left side $(\mathrm{p}=0.020)$; regarding location and size, the central lesions were larger than the peripheral ones $(51.7$ and $17.4 \mathrm{~mm}$ respectively, $\mathrm{p}<0.01$ ). The bivariate regression analysis showed a direct correlation between the SUVmax and the clinical tumor size: Spearman's rho 0.552, $R^{2} 0.30, p=0,004$ (Figure 1). Of the 11 tumors with a diameter $<20 \mathrm{~mm}$, only 2 (18\%) showed a SUVmax $>2.5$ (2.8 and 4.6), and $50 \%$ of the tumors with a diameter $>30 \mathrm{~mm}$ showed a SUVmax $>2.5(n=3 / 6)$. A similar significant correlation was found when pathological size was considered in the regression analysis and compared to SUVmax. No further correlations were found between the SUVmax and the other clinical, radiological and pathological variables.

Follow-up was complete for all the patients, with a mean of 43 months (range 12-74 months). None of the patients had recurrent carcinoid disease or died during the follow-up.

\section{Discussion}

Bronchial carcinoids enter the differential diagnosis of SPN or mass. On the CT scan they typically present as nodules or masses with an ovoid/round shape and smooth

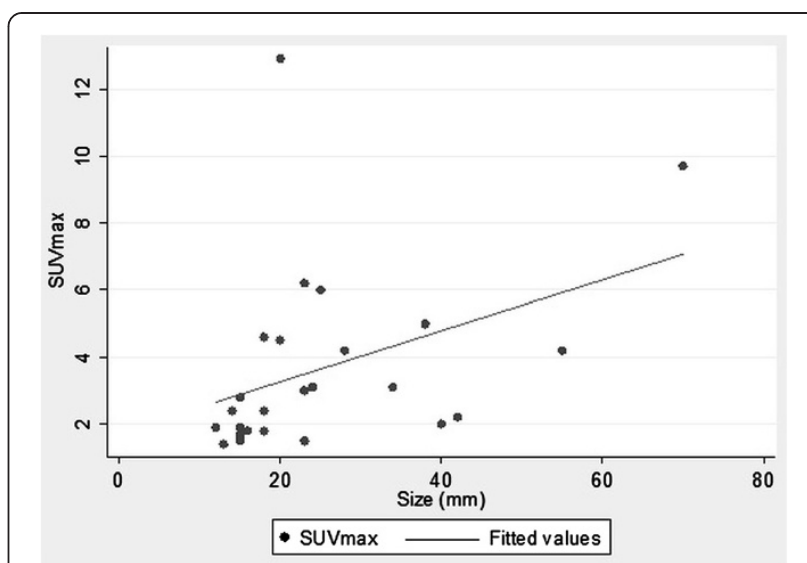

Figure 1 The linear correlation model between the SUVmax and tumor diameter shows a significant direct correlation.

margins [3]. However, also hamartomas, metastases, infectious/inflammatory lesions or, in some cases, lung carcinoma, can present this radiological aspect. On the other hand, carcinoids can present with spiculated margins and irregular shape, although less commonly (8/25 patients, $32 \%$ in our series).

Because carcinoids express somatostatin receptors on their surface, functional imaging with radiolabeled somatostatin analogues is the examination of choice $[11,13]$. In clinical practice, however, when a SPN or mass is detected by $\mathrm{CT}$ scan, the diagnostic work-up is frequently completed using FDG-PET, or, preferably, integrated FDG$\mathrm{PET} / \mathrm{CT}$, if available [14]. But pulmonary carcinoids have been traditionally described as tumors with low grade of FDG uptake, most likely because of their low metabolism and slow growth. Therefore, FDG-PET has been commonly understood to have a reduced sensitivity for carcinoids and a limited role in the diagnostic work-up of these lesions. This conviction originated from the first work on FDG-PET and carcinoids by Erasmus in 1998 [8], who reported on 7 carcinoids, of which 6 were hypometabolic (86\%) and thus erroneously considered benign. Later studies, however, did not confirm those findings. Wartski described two carcinoids with intense FDG uptake (SUVmax 4.8 and 10.6) [15]. Kruger reported 13 carcinoids of which $54 \%$ were hypermetabolic (SUVmax >2.5) [16]. Daniels studied 16 patients and found a sensitivity for FDG-PET detection of carcinoids of $75 \%$ [9]. Chong found that the uptake in 3 of 7 carcinoids (43\%) was higher than the mediastinal uptake and they were considered positive on the PET/CT scan [10]. Kayani reported that 9 of 13 patients with carcinoids (69\%) showed significant FDG uptake and were considered to have malignant lesions [11]. Jindal found an overall detection rate by FDG-PET/CT of $70 \%$ in a series of 20 carcinoids [13]. 
The method of assessment of the nodule uptake is of utmost importance for an adequate evaluation of the accuracy of FDG-PET for carcinoids. The sensitivity of FDG-PET for carcinoids was generally investigated on the basis of a visual assessment or of a SUVmax cutoff. Regarding the visual assessment, the PET scan results were tipically interpreted as positive when the nodule activity was greater than the background mediastinal pool activity (8-10). When the SUVmax was used to define the PET positivity, a cut-off of 2.5 was commonly applied $[11,13,16]$. Both methods of assessment of nodule activity are derived from the evaluation of lung carcinoma [17]. But carcinoids, especially the typical forms, show a lower metabolic activity with respect to lung carcinoma, therefore, a different method should be applied for the evaluation of FDG uptake. We hypothesized that it may be more appropriate to compare the nodule activity with the normal lung and not with the mediastinal blood pool activity, so that the majority of the nodules can be easily visualized, as noted by Erasmus [8]. The evaluation of SUVmax is mandatory with this method of assessment. However, the cut-off value of 2.5 appears to be no longer adequate, especially for TCs. As reported by other authors $[8,11,13,14]$, also in our series a number of TCs showed a SUVmax $<2.5$ (12 patients of 24,50\%). If a cut-off of 1.5 was fixed, no false-negative results would have been present in the series by Erasmus ( $0 / 6$ patients) [8] and Kayani (0/11) [11] and reduced false-negative rates of $23 \%, 8 \%$ and $4 \%$ would have been found in the series by Jindal [13] and Kruger [16] and in our series, respectively.

FDG uptake of carcinoids was found to be related to the histologic type, proliferation rate and size of the lesion. Several authors found that ACs showed a significantly higher FDG uptake than TC and in many cases this uptake was comparable to that of lung carcinoma [9-11,13,15]. This is because ACs show high proliferation rates compared to TCs. Our series included 24 TCs (96\%) and one AC, thus an analysis based on the histologic subtype was not feasible. Regarding the tumor size, for lung carcinoma it is well known that the intensity of the FDG uptake can be directly correlated to the tumor dimensions [18,19]. Erasmus [8] and Kruger [16] demonstrated that the SUVmax was directly related to the tumor diameter also for bronchial carcinoids. Also Daniels reported the correlation between the FDG uptake and the size of the neoplasm but the SUVmax was not calculated in his work [9]. In our series we found a direct correlation between the SUVmax and the size of the tumor, when both the clinical and pathological diameters were investigated (Figure 1). This relationship may partly explain the variability in the FDG uptake in our series of TCs (SUVmax range 1.4 - 12.9), although some exceptions are present (Figures 2 and 3). We did not find any association between the SUVmax and other tumor features, such as location, shape or margins; similar associations have not been reported in the literature.

Some authors found a correlation between the staging of lung carcinoids by FDG-PET and surgical staging $[9,10]$. In our series, FDG-PET was negative for nodal metastases in all patients. This finding correlated well with the pathological findings, because all the patients resulted pN0, except for the AC patient, who had pN1 disease. However, because our series lack of a comparison group of $\mathrm{N}+$ patients, conclusions regarding PET utility for nodal disease remain uncertain.

In our study, 4 patients received a preoperative diagnosis of TC by fiberoptic bronchoscopy. It is generally accepted that FDG-PET is not mandatory in the clinical evaluation of patients with biopsy-proven pulmonary carcinoid $[9,11]$ and we agree with this statement. Our series includes patients with biopsy-proven TC only because they underwent PET/CT before bronchoscopy during their staging work-up. The disproportionate number of peripheral carcinoids in our study population reflects the clinical policy to consider that PET/CT is not necessary in cases of a preoperative diagnosis of bronchial carcinoid, which is usually obtained in central forms by bronchoscopy.

Long-term prognosis for patients affected by $\mathrm{TC}$ is favourable, with 5-year survival rates higher than 95\% after radical surgical resection [20]. This rate is consistent with our findings, which showed no deaths or recurrences of carcinoid disease at follow-up.

In our study, all the lesions were clearly detectable as SPNs on helical CT. In many cases, ovoid/round shape and regular margins suggested a carcinoid, and the main

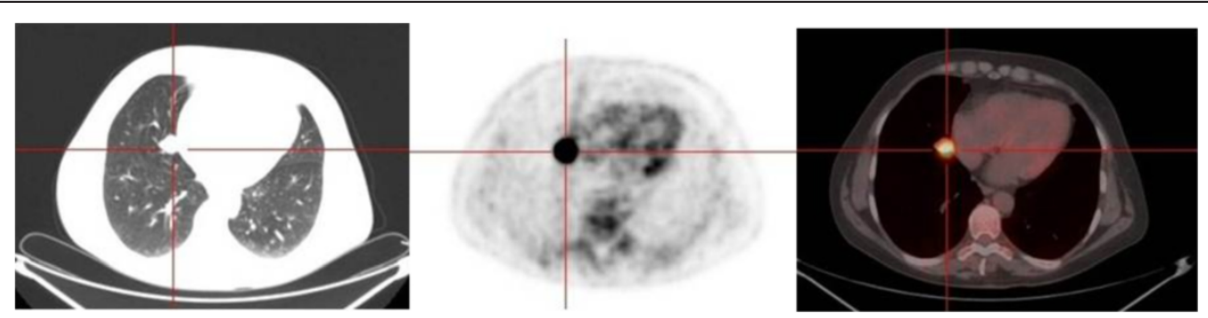

Figure 2 The integrated FDG-PET/CT reveals a small TC $(20 \mathrm{~mm}$ ) demonstrating high uptake (SUVmax 12.9). From left to right: CT, PET and fused image. 


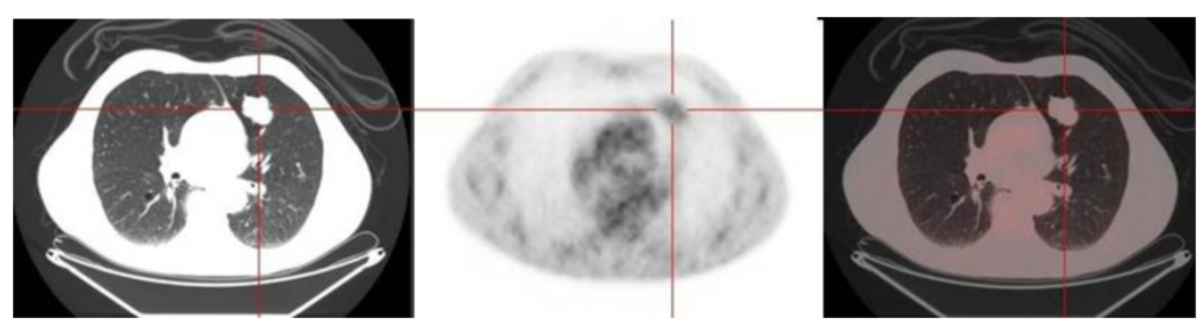

Figure 3 The integrated FDG-PET/CT reveals a large TC $(42 \mathrm{~mm}$ ) demonstrating low uptake (SUVmax 2.2). From left to right: CT, PET and fused image.

differential diagnosis was with hamartoma or other benign lesions. In a minority of cases, spiculated margins and irregular shape led to inclusion of lung carcinoma in the differential diagnosis. All of these lesions showed FDG uptake, therefore none was considered typically benign, and we proceeded to surgical resection in all cases. For the lesions with a lower uptake, a diagnosis of hamartoma was considered unlikely, because the FDG uptake is exceptional for hamartomas [21,22]; infections or inflammatory lesions remained in the differential diagnosis but they were considered less probable than carcinoids after a careful examination of the patient. Regarding the lesions with a higher uptake, a diagnosis of lung carcinoma, particularly a low-grade adenocarcinoma, was taken into account; in these latter cases, a further unconfirmed suspicion of carcinoid would not have affected the adequacy of the surgical indication.

The assessment of the nodule characteristic was performed analysing the images of enhanced helical CT-scan rather than $\mathrm{CT}$ images of the integrated PET/CT scan. The acquisition parameters of CT scan of the integrated $\mathrm{PET} / \mathrm{CT}$ are not adequate for a careful assessment of the nodule features, especially for small lesions. Moreover, the enhanced helical CT scan is usually the first approach to the SPN following the chest-x-ray and, therefore, is available for all patients.

The main limitation of this study is its retrospective nature. The data only included nodules that were resected and may underestimate the number of PET false-negative lesions.

\section{Conclusions}

We believe that for cases in which an SPN with an ovoid/round shape and smooth margins on the CT scan reveals an FDG uptake higher than that of the normal lung and with a SUVmax value $>1-1.5$, a bronchial carcinoid should be considered and surgical resection or at least a biopsy of the lesion should be recommended.

Further studies comparing the PET/CT findings of carcinoids, hamartomas, low-grade adenocarcinomas and infectious/inflammatory lesions should be advocated.

\section{Abbreviations}

18F-FDG-PET: 18F-fluorodeoxyglucose positron emission tomography; AC: Atypical carcinoids; CT: Computed tomography; ROI: Region of interest; SD: Standard deviation; SPN: Solitary pulmonary nodule; SUV: Standardized uptake value; TC: Typical carcinoids.

\section{Competing interests}

The authors declare that they have no competing interests.

\section{Authors' contribution}

AS conceived of the study, participated in its design and drafted the manuscript. AF, AC and LM performed the SUVmax analysis, helped to draft the manuscript and drafted Figures 2 and 3. JN and CP retrieved the data and created the database. BA retrieved the data and performed the language revision. SK performed the statistical analysis and drafted Figure 1. UM participated in conceiving the study and its design and coordinated the study. All authors read and approved the final manuscript.

\section{Author details}

'Department of Thoracic Surgery, University of Modena and Reggio Emilia, Modena, Italy. ${ }^{2}$ Department of Nuclear Medicine, University of Modena and Reggio Emilia, Modena, Italy. ${ }^{3}$ Department of Biostatistics, University of Modena and Reggio Emilia, Modena, Italy.

Received: 7 August 2013 Accepted: 25 November 2013

Published: 4 December 2013

\section{References}

1. Modlin IM, Lye KD, Kidd M: A 5-decade analysis of 13.715 carcinoid tumors. Cancer 2003, 97:934-959.

2. Brambilla E, Travis WD, Colby TV, Corrin B, Shimosato Y: The new World Health Organization classification of lung tumors. Eur Respir J 2001, 18:1059-1068

3. Davila DG, Dunn WF, Tazelaar HD, Pairolero PC: Bronchial carcinoid tumors. Mayo Clin Proc 1993, 68:795-803.

4. Buchmann I, Henze M, Engelbrecht S, Eisenhut M, Runz A, Schäfer M, Schilling T, Haufe S, Herrmann T, Haberkorn U: Comparison of ${ }^{68} \mathrm{Ga}$-DOTATOC PET and ${ }^{11}$ In-DTPAOC (OctreoScan) SPECT in patients with neuroendocrine tumors. Eur J Nucl Med Mol Imaging 2007, 34:1617-1626.

5. Antunes P, Ginj M, Zhang H, Waser B, Baum RP, Reubi JC, Maecke H: Are radiogallium-labeled DOTA-conjugated somatostatin analogues superior to those labelled with other radiometals? Eur I Nucl Med Mol Imaging 2007, 34:982-993.

6. Gould MK, Maclean CC, Kuschner WG, Rydzak CE, Owens DK: Accuracy of positron emission tomography for diagnosis of pulmonary nodules and mass lesions: a meta-analysis. JAMA 2001, 285:914-924.

7. Yi CA, Lee KS, Kim BT, Choi JY, Kwon OJ, Kim H, Shim YM, Chung MJ: Tissue characterization of solitary pulmonary nodule: comparative study between helical dynamic CT and integrated PET/CT. J Nucl Med 2006, 47:443-450.

8. Erasmus JJ, MCAdams HP, Patz EF, Coleman RE, Ahuja V, Goodman PC: Evaluation of primary pulmonary carcinoid tumors using FDG PET. AJR 1998, 170:1369-1373.

9. Daniels CE, Lowe VJ, Aubry MC, Allen MS, Jett JR: The utility of fluorodeoxyglucose positron emission tomography in the evaluation of 
carcinoid tumors presenting as pulmonary nodules. Chest 2007, 131:255-260.

10. Chong S, Lee KS, Kim BT, Choi JY, Yi CA, Chung MJ, Oh DK, Lee JY: Integrated PET/CT of pulmonary neuroendocrine tumors: diagnostic and prognostic implications. AJR 2007, 188:1223-1331.

11. Kayani I, Conry BG, Groves AM, Win T, Dickson J, Caplin M, Bomanji JB: A comparison of ${ }^{68} \mathrm{Ga}$-DOTATATE and ${ }^{18} \mathrm{~F}$-FDG PET/CT in pulmonary neuroendocrine tumors. J Nucl Med 2009, 50:1927-1932.

12. Travis WD, Brambilla E, Muller-Hermelink HK, et al (Eds): Tumours of the Lung, Pleura, Thymus and Heart. Pathology and Genetics. WHO Classification of Tumours. Lyon: IARC Press; 2004

13. Jindal T, Kumar A, Venkitaraman B, Meena M, Kumar R, Malhotra A, Dutta R: Evaluation of the role of $\left[{ }^{18} \mathrm{~F}\right] \mathrm{FDG}-\mathrm{PET} / \mathrm{CT}$ and $\left[{ }^{68} \mathrm{Ga}\right] \mathrm{DOTATOC}-\mathrm{PET} / \mathrm{CT}$ in differentiating typical and atypical pulmonary carcinoids. Cancer Imaging 2011, 11:70-75.

14. Lardinois D, Weder W, Hany TF, Kamel EM, Korom S, Seifert B, von Schulthess GK, Steinert HC: Staging of non-small cell lung cancer with integrated positronemission tomography and computed tomography. N Engl J Med 2003, 348:2500-2507.

15. Wartski M, Alberini JL, Leroy-Ladurie F, De Montpreville V, Nguyen C, Corone C, Dartevelle P, Pecking AP: Typical and atypical bronchopulmonary carcinoid tumors on FDG-PET/CT imaging. Clin Nucl Med 2004, 29:752-753.

16. Kruger K, Buck AK, Blumstein NM, Pauls S, Schelzig H, Kropf C, Schumann C, Mottaghy FM, Hombach V, Reske SN: Use of integrated FDG-PET/CT imaging in pulmonary carcinoid tumors. J Int Med 2006, 260:545-550.

17. Kim RH, Kim DJ, Lee WW, Jheon S, Sung SW: The significance of maximum standardized uptake values in patients with stage I pulmonary adenocarcinoma. Eur J Cardiothorac Surg 2009, 35:712-717.

18. Cerfolio RJ, Bryant AS, Ohja B, Bartolucci AA: The maximum standardized uptake values on positron emission tomography of a non-small cell lung cancer predict stage, recurrence and survival. J Thorac Cardiovasc Surg 2005, 130:151-159.

19. Casali C, Cucca M, Rossi G, Barbieri F, lacuzio L, Bagni B, Morandi U: The variation of prognostic significance of maximum standardized uptake value of (18 F)-fluoro-2-deoxy-glucose positron emission tomography in different histological subtypes and pathological stages of surgically resected non-small cell lung carcinoma. Lung Cancer 2012, 69:187-193.

20. Ferguson MK, Landreneau RJ, Hazelrigg SR, Altorki NK, Naunheim KS: Long-term outcome after resection of bronchial carcinoid tumors. Eur J Cardiothorac Surg 2000, 18:156-161.

21. De Cicco C, Bellomi M, Bartolomei M, Carbone G, Pelosi G, Veronesi G, DePas T, Spaggiari L, Paganelli G: Imaging of lung hamartomas by multidetector computed tomography and positron emission tomography. Ann Thorac Surg 2008, 86:1769-1772.

22. Himpe U, Deroose CM, Leyn PD, Verbeken E, Vansteenkiste J: Unexpected slight fluorodeoxyglucose-uptake on positron emission tomography in a pulmonary hamartoma. J Thorac Oncol 2009, 4:107-108.

doi:10.1186/1749-8090-8-223

Cite this article as: Stefani et al:: Integrated FDG-PET/CT imaging is useful in the approach to carcinoid tumors of the lung. Journal of Cardiothoracic Surgery 2013 8:223.

\section{Submit your next manuscript to BioMed Central and take full advantage of:}

- Convenient online submission

- Thorough peer review

- No space constraints or color figure charges

- Immediate publication on acceptance

- Inclusion in PubMed, CAS, Scopus and Google Scholar

- Research which is freely available for redistribution 